\title{
Wave Propagation in a Compressible Ionosphere. Part II
}

\author{
S. R. Seshadri \\ Contribution From Applied Research Laboratory, Sylvania Electronic Systems, a Division of Sylvania \\ Electric Products, Inc., Waltham 54, Mass.
}

(Received July 15, 1964)

\begin{abstract}
In this part of the paper, the higher order approximations to the general dispersion equation are considered. It is shown first that the results of the first approximation are also found to be valid in the neighborhood of $\Omega=0$, even though the phase velocity goes to zero for that value of $\Omega$. The second approximation which gives correctly the phase velocities of the order of the acoustic velocity $a$, in the electron gas or lower and the third approximation which yields the phase velocities of the order $\sqrt{a C_{0}^{\prime}}$ are worked out. A synthesis of the results of the first three approximations yields the dispersion curves for the three independent modes which propagate in an electron plasma.

The dispersion curves for the three modes obtained as a result of the first three approximations are not valid in the axial boundary layer, where the dispersion is found to undergo rapid changes in the close nieghborhood of the frequencies $\Omega=R$ and $\Omega=1$. Using the boundary layer techniques, the dispersion equations in the axial boundary layer, for the frequencies in the close neighborhood of $\Omega=R$ and $\Omega=1$ are obtained and analyzed. The boundary layer analysis clarifies the mechanism of coupling between the purely longitudinal plasma waves and the purely transverse electromagnetic waves. With the knowledge of the above coupling that takes place in the axial boundary layer, the three independent modes may be properly designated using a procedure which is found to be uniformly valid for all directions of propagation and the inconsistency found in the magneto-ionic approximation is removed.
\end{abstract}

\section{Introduction}

The characteristics of a plane wave propagating in an unbounded, collision-free, compressible electron plasma along a direction which makes an arbitrary angle with that of the static magnetic field are studied in this paper. In part I of this paper, the cubic equation which specifies the square of the phase velocity of the plane wave was derived and was shown to factor out into a linear and a quadratic equation for the two special cases of propagation, namely those along and across that of the external magnetic field. This feature enabled the dispersion relations for the above mentioned special cases to be obtained exactly without any difficulty. For the general case of propagation in an arbitrary direction with respect to that of the static external magnetic field, the exact dispersion equation lent itself to be approximated by a quadratic equation in the situation where only the phase velocities of the order of the velocity $C_{0}$ of the electromagnetic waves in free space or higher are sought. The analysis of the dispersion equation obtained in the first approximation yielded zero values for the phase velocity for the three frequencies, $\Omega=0, \Omega_{3}$ and $\Omega_{4}$. Consequently the validity of the first approximation becomes questionable in the neighborhood of the above three frequencies.
In this part of the paper, it is shown that the first approximation is accurate in the neighborhood of the frequency $\Omega=0$ despite the fact that the phase velocity becomes zero for that frequency. A second approximation to the dispersion equation is obtained which gives accurately the phase velocities of the order of the acoustic velocity $a$ in the electron gas or lower. The second approximation yields a linear equation and its analysis shows that the phase velocity becomes infinite for the same two frequencies $\Omega=\Omega_{3}$ and $\Omega_{4}$ for which the first approximation yielded zero values. The first two approximations are obviously not accurate in the neighborhood of the two frequencies $\Omega=\Omega_{3}$ and $\Omega_{4}$. A third approximation which is valid in the neighborhood of the two frequencies $\Omega=\Omega_{3}$ and $\Omega_{4}$ is obtained and it shows that the phase velocities for those two frequencies are of the order $\sqrt{a C_{j}}$. Moreover, the results of the third approximation are found to merge continuously with those of the first two approximations, thus yielding the overall dispersion curves for the three independent modes that propagate in an electron plasma.

The dispersion curves for the three modes obtained as a result of the first three approximations, change as the direction of propagation is changed and attain the known values for the propagation across the static magnetic field but not for the case along it. The reason for this behavior is shown to be due 
to the fact that in a small region about the direction of the static magnetic field, called as the axial boundary layer, the dispersion in the neighborhood of the two frequencies $\Omega=R$ and $\Omega=1$ changes very rapidly. By employing boundary layer techniques the dispersion equations in the axial boundary layer in the close neighborhood of the frequencies $\Omega=R$ and $\Omega=1$ are derived and analyzed. This analysis enables the proper understanding of the mechanism of coupling between the purely longitudinal plasma waves and the purely transverse electromagnetic waves for the case of propagation other than that parallel to the static magnetic field. Once the mechanism of coupling is understood, it is possible to designate the three independent modes that propagate in an electron plasma using a procedure which is uniformly valid for all directions of propagation and the inconsistency found in the magneto-ionic approximation is no longer found to exist.

\section{Higher Order Approximations for the Re- gion Exterior to the Axial Boundary Layer}

It will be shown now that even though the phase velocity becomes zero for $\Omega=0$, the approximation (25), which was derived from (24a) on the condition that the phase velocity is of the order $C_{0}$ or higher, is still valid in the neighborhood of $\Omega=0$. In the limit of $\Omega$ tending to zero, (25) together with $(24 \mathrm{~b}, \mathrm{c})$ become

where

$$
\lambda^{4}+\bar{A}_{1}^{(1)} \lambda^{2}+\bar{A}_{2}^{(1)}=0
$$

$$
\begin{aligned}
& \bar{A}_{1}^{(1)}=\Omega^{2} C_{0}^{2}\left[2+R^{2}\left(1+l^{2}\right)\right] \\
& \bar{A}_{2}^{(1)}=-\Omega^{2} C_{0}^{4} R^{2} l^{2} .
\end{aligned}
$$

If $\Omega$ is of the order $a^{2} / C_{0}^{2}$, the solution of (30a), on omitting terms of the order $a^{2} / C_{0}^{2}$ in comparison with unity, is obtained as

$$
\lambda^{2}=\sqrt{-\bar{A}_{2}^{(1)}}=\Omega C_{0}^{2} R l .
$$

For $\Omega$ of the order $a^{2} / C_{0}^{2}$, (31) shows that the phase velocity is of the order $a$. In the limit of $\Omega$ tending to zero, the exact dispersion equation, after omitting the terms of the order $a^{2} / C_{0}^{2}$ in comparison with unity, becomes

$$
\lambda^{6}+\bar{A}_{1}^{(1)} \lambda^{4}+\bar{A}_{2}^{(1)} \lambda^{2}+\bar{A}_{3}^{(1)}=0
$$

where $\bar{A}_{1}{ }^{(1)}$ and $\bar{A}_{2}{ }^{(1)}$ are the same as in $(30 \mathrm{~b})$ and (30c) respectively and

$$
\bar{A}_{3}^{(1)}=-\Omega^{4} C_{0}^{4} a^{2} R^{2} l^{2} .
$$

When the phase velocity $\lambda$ is of the order $a$ and $\Omega$ is of the order $a^{2} / C_{0}^{2}$, on omitting the terms of the order

\footnotetext{
1 The equations and the figures in parts I and II of this paper are numbered
consecutively for the sake of convenience.
}

$a^{2} / C_{0}^{2}$ and $a^{4} / C_{0}^{4}$ in comparison with unity, it is found that only the first and the third terms, which are of the order $a^{6}$, need be retained on the left side of (32a) with the result

$$
\lambda^{2}=\sqrt{-\bar{A}_{2}^{(1)}} .
$$

Note that (33) obtained from the exact dispersion equation (24a) is the same as (31) which was obtained from the approximate dispersion equation (25). Therefore, the validity of (25) in the neighborhood of $\Omega=0$ is not impaired even though the phase velocity goes to zero for that frequency. It is to be noted with the help of (31) that the phase velocity goes to zero as $\Omega^{1 / 2}$ in the limit of $\Omega$ tending to zero.

If the phase velocity is of the order $a$, it is seen from (24a) that the fourth and the sixth terms on the left side of (24a) are of the order $C_{0}^{4} a^{2}$ and the remaining terms are at least of the order $a^{2} / C_{0}^{2}$ lower than these two terms. Hence, only the fourth and the sixth terms need be retained with the following result

$$
\begin{aligned}
\lambda^{2} & =-\frac{A_{3}^{(1)}}{A_{2}^{(1)}}=\frac{a^{2} \Omega^{2}\left(\Omega^{2}-R^{2} l^{2}\right)}{\Omega^{4}-\Omega^{2}\left(1+R^{2}\right)+R^{2} l^{2}} \\
& =\frac{a^{2} \Omega^{2}\left(\Omega^{2}-R^{2} l^{2}\right)}{\left(\Omega^{2}-\Omega_{3}^{2}\right)\left(\Omega^{2}-\Omega_{4}^{2}\right)} .
\end{aligned}
$$

Since $\Omega_{3}^{2}<R^{2} l^{2}<\Omega_{4}^{2}$, (34) gives real phase velocities only for $\Omega_{3}^{2}<\Omega^{2}<R^{2} l^{2}$ and $\Omega_{4}^{2}<\Omega^{2}<\infty$. In figures 6 a and $b$, the dotted portion shows the real phase velocity given by (34). In the second approximation, the phase velocity diagram is seen to have two branches, one of which goes to zero for $\Omega^{2}=R^{2} l^{2}$ and the other asymptotically approaches the acoustic velocity $a$ in the electron gas as $\Omega$ tends to infinity. Since the approximation (34) is valid strictly only for the phase velocities of the order of $a$ or lower and since the phase velocity given by (34) becomes infinite for $\Omega=\Omega_{3}$ and $\Omega=\Omega_{4}$, it is obvious that (34) also, is not a valid approximation in the neighborhood of the frequencies $\Omega=\Omega_{3}$ and $\Omega=\Omega_{4}$.

A third approximation to the exact dispersion equation (24a) can be obtained in the following way to yield phase velocities which are reasonably accurate for frequencies in the neighborhood of $\Omega=\Omega_{3}$ and $\Omega=\Omega_{4}$. If the phase velocity is of the order $\sqrt{a C_{0}}$, it is found that the terms on the lefthand side of (24a) are of the order

$$
\begin{aligned}
& a C_{0}^{5}\left(\frac{a}{C_{0}}\right)^{2}, a C_{0}^{5}\left(\frac{a}{C_{0}}\right), a C_{0}^{5}\left(\frac{a}{C_{0}}\right)^{3}, \\
& \qquad a C_{0}^{5}, a C_{0}^{5}\left(\frac{a}{C_{0}}\right)^{2} \text {, and } a C_{0}^{5}\left(\frac{a}{C_{0}}\right),
\end{aligned}
$$

respectively. Hence only the second, the fourth, and the sixth terms need be retained with the result

$$
\lambda^{4}+\frac{A_{20}^{(1)}}{A_{10}^{(1)}} \lambda^{2}+\frac{A_{30}^{(1)}}{A_{10}^{(1)}}=0 .
$$


The solution of (35) is

$$
\lambda^{2}=-\frac{A_{20}^{(1)}}{2 A_{10}^{(1)}} \pm \sqrt{\left(\frac{A_{20}^{(1)}}{2 A_{10}^{(1)}}\right)^{2}-\frac{A_{30}^{(1)}}{A_{10}^{(1)}}}
$$

From figure 4, it is evident that $A_{10}^{(1)}<0$ in the neighborhood of $\Omega^{2}=\Omega_{3}^{2}$ and that $A_{10}^{(1)}>0$ in the neighborhood of $\Omega^{2}=\Omega_{4}^{2}$. Also from (24c) and the fact that $\Omega_{3}^{2}<R^{2} l^{2}<\Omega_{4}^{2}$, it is clear that $A_{30}^{(1)}>0$ in the neighborhood of $\Omega^{2}=\Omega_{3}^{2}$ and $A_{30}^{(1)}<0$ in the neighborhood of $\Omega^{2}=\Omega_{4}^{2}$. Hence $A_{30}^{(1)} / A_{10}^{(1)}<0$ for the value of $\Omega$ near both $\Omega_{3}$ and $\Omega_{4}$. Therefore

$$
\left|\sqrt{\left(\frac{A_{20}^{(1)}}{2 A_{10}^{(1)}}\right)^{2}-\frac{A_{30}^{(1)}}{A_{10}^{(1)}}}\right|>\left|\frac{A_{20}^{(1)}}{2 A_{10}^{(1)}}\right|
$$

and only the upper sign in (36) will lead to a real value for the phase velocity and, consequently, the lower sign may be disregarded.

When $\Omega^{2}$ is sufficiently removed from either $\Omega_{3}^{2}$ or $\Omega_{4}^{2}$, it is found with the help of $(24 \mathrm{~b}-\mathrm{d})$ that $A_{30}^{(1)} /$ $A_{10}^{(1)}$ is of the order $a^{2} / C_{0}^{2}$ less than $\left(A_{20}^{(1)}\right)^{2} /\left(2 A_{10}^{(1)}\right)^{2}$. The square root in (36) may, therefore, be expanded yielding the following result:

$$
\lambda^{2}=-\frac{A_{20}^{(1)}}{2 A_{10}^{(1)}}+\left|\frac{A_{20}^{(1)}}{2 A_{10}^{(1)}}\right|-\frac{A_{30}^{(1)} A_{10}^{(1)}}{\left|A_{20}^{(1)}\right|\left|A_{10}^{(1)}\right|} .
$$

For $\Omega^{2}>\Omega_{3}^{2}$, it is seen from figure 4 that $A_{10}^{(1)}<0$ and $A_{20}^{(1)}<0$. Similarly for $\Omega^{2}>\Omega_{4}^{2}, A_{10}^{(1)}>0$, and $A_{20}^{(1)}>0$. For $\Omega^{2}$ sufficiently greater than $\Omega_{3}^{2}$ or $\Omega_{4}^{2}, A_{20}^{(1)} /$ $A_{10}^{(1)}>0$ and, therefore, (37) becomes

$$
\lambda^{2}=-\frac{A_{30}^{(1)}}{A_{20}^{(1)}} .
$$

It is found, on comparing (34) and (38), that the phase velocity (38) obtained from the third approximation becomes identical with that given by the second approximation for $\Omega^{2}$ sufficiently greater than $\Omega_{3}^{2}$ or $\Omega_{4}^{2}$.

For $\Omega^{2}<\Omega_{3}^{2}, A_{10}^{(1)}<0$ and $A_{20}^{(1)}>0$. Also for $\Omega^{2}<\Omega_{4}^{2}$, $A_{10}^{(1)}>0$ and $A_{20}^{(1)}<0$. Hence for $\Omega^{2}<\Omega_{3}^{2}$, and $\Omega^{2}<\Omega_{4}^{2}$, on omitting the terms of the order $a^{2} / C_{0}^{2}$ in comparison with unity (37) becomes

$$
\lambda^{2}=-\frac{A_{20}^{(1)}}{A_{10}^{(1)}}
$$

As $\Omega$ approaches from below the close vicinity of either $\Omega_{3}$ or $\Omega_{4}$, it is found with the help of (24c) that the second term inside the radical in (26) becomes very much smaller in comparison with the first term, enabling the radical in (26) to be expanded yielding the following result:

$$
\lambda_{e, 0}^{2}=-\frac{A_{10}^{(1)}}{2 A_{0}} \pm \frac{\left|A_{10}^{(1)}\right|}{2 A_{0}} \mp \frac{A_{20}^{(1)}}{\left|A_{10}^{(1)}\right|} .
$$

The upper and the lower signs in (40) correspond respectively to the extraordinary and the ordinary electromagnetic modes. For the $O^{\mathrm{II}}$ mode and for $\Omega$ slightly less than $\Omega_{3}$, figure 4 shows that $A_{10}^{(1)}<0$ and this reduces (40) to

$$
\lambda_{0}^{2}=-\frac{A_{20}^{(1)}}{A_{10}^{(1)}} .
$$

Since, as seen from figure $4, A_{20}^{(1)}>0$ for $\Omega^{2}<\Omega_{3}^{2}$, (41a) leads to real values for the phase velocity. In the same manner, it is seen from figure 4 that $A_{10}^{(1)}>0$ for the $e^{\mathrm{II}}$ mode and hence for $\Omega$ slightly less than $\Omega_{4}$ is found to yield

$$
\lambda_{e}^{2}=-\frac{A_{20}^{(1)}}{A_{10}^{(1)}}
$$

Using figure 4 , it is found that $A_{20}^{(1)}<0$ for $\Omega^{2}<\Omega_{4}^{2}$ and therefore $(41 \mathrm{~b})$ gives real values for the phase velocity. A comparison of (39) with $(41 \mathrm{a}, \mathrm{b})$ shows that the phase velocities obtained from the first approximation for $\Omega^{2}$ very close to but less than either $\Omega_{3}^{2}$ or $\Omega_{4}^{2}$ are identical with those obtained from the third approximation.

Also the third approximation (35), as seen from (36) and the argument following it, gives the following expression for the phase velocity for $\Omega^{2}=\Omega_{3}^{2}$ and $\Omega^{2}=\Omega_{4}^{2}$ :

$$
\lambda^{2}=\sqrt{\frac{-A_{30}^{(1)}}{A_{10}^{(1)}}} .
$$

Since $A_{30}^{(1)} / A_{10}^{(1)}<0$ for $\Omega^{2}=\Omega_{3}^{2}$ and $\Omega^{2}=\Omega_{4}^{2}$, (42) is seen to yield real values for the phase velocities for $\Omega^{2}=\Omega_{3}^{2}$ and $\Omega^{2}=\Omega_{4}^{2}$. In addition, with the help of $(24 \mathrm{~b}, \mathrm{~d}),(42)$ is found to give a phase velocity of the order $\sqrt{a C_{0}}$ for $\Omega^{2}=\Omega_{3}^{2}$ and $\Omega^{2}=\Omega_{4}^{2}$. Consequently, the third approximation (35) which was derived from the exact dispersion equation (24a) on the condition that the phase velocity is of the order $\sqrt{a C_{0}}$, is certainly very valid in the close vicinities of both $\Omega^{2}=\Omega_{3}^{2}$ and $\Omega^{2}=\Omega_{4}^{2}$.

The synthesis of the results obtained in the three approximations shows that the dispersion curves for the $O^{\mathrm{I}}$ and the $e^{\mathrm{I}}$ modes obtained in the first approximation and therefore strictly from the application of the magneto-ionic theory are practically unaltered by the two higher order approximations which, in essence, include the effect of the compressibility of the medium. However, the $O^{\mathrm{II}}$ and the $e^{\mathrm{II}}$ modes are affected in the following manner. The $O^{\mathrm{II}}$ mode as given in the first approximation, instead of going to zero for $\Omega=\Omega_{3}$, continues and goes to zero for $\Omega^{2}=R^{2} l^{2}$. The dispersion for the $O^{\mathrm{II}}$ mode is given by (29) for $0 \leq \Omega^{2}<\Omega_{3}^{2}$, by (36) in the close neighborhood of $\Omega^{2}=\Omega_{3}^{2}$ and by (34) for $\Omega_{3}^{2}<\Omega^{2} \leq R^{2} l^{2}$. On the other hand, the $e^{\text {II }}$ mode as obtained in the first approximation is modified by the higher order approximations in such a manner that the phase velocity instead of going to zero for $\Omega=\Omega_{4}$, levels off to the value of the acoustical velocity $a$ in the electron gas and thus continues up to $\Omega=\infty$ for which value the phase velocity is exactly equal to $a$. The dispersion for the modified $e^{\mathrm{II}}$ mode is given by (29) for $\Omega_{1}^{2} \leq \Omega^{2}<\Omega_{4}^{2}$, by (36) in 
the close neighborhood of $\Omega^{2}=\Omega_{4}^{2}$ and by (34) for $\Omega_{4}^{2}<\Omega^{2}<\infty$. The figures $6 \mathrm{a}, \mathrm{b}$ are respectively redrawn in figures $7 \mathrm{a}, \mathrm{b}$ wherein the third approximation (36) is included for the phase velocities in the close neighborhood of $\Omega^{2}=\Omega_{3}^{2}$ and $\Omega^{2}=\Omega_{4}^{2}$ and thus the complete dispersion curves for the arbitrary direction of propagation of the plane wave are obtained.

It is convenient to redesignate these modes in such a way as to fit in with the existing nomenclature for these modes for the two special directions of propagation, namely those along and across the static magnetic field. The $O^{\mathrm{r}}$ mode is obviously the modified ordinary electromagnetic mode $[\mathrm{MEM}(O)]$ since it is

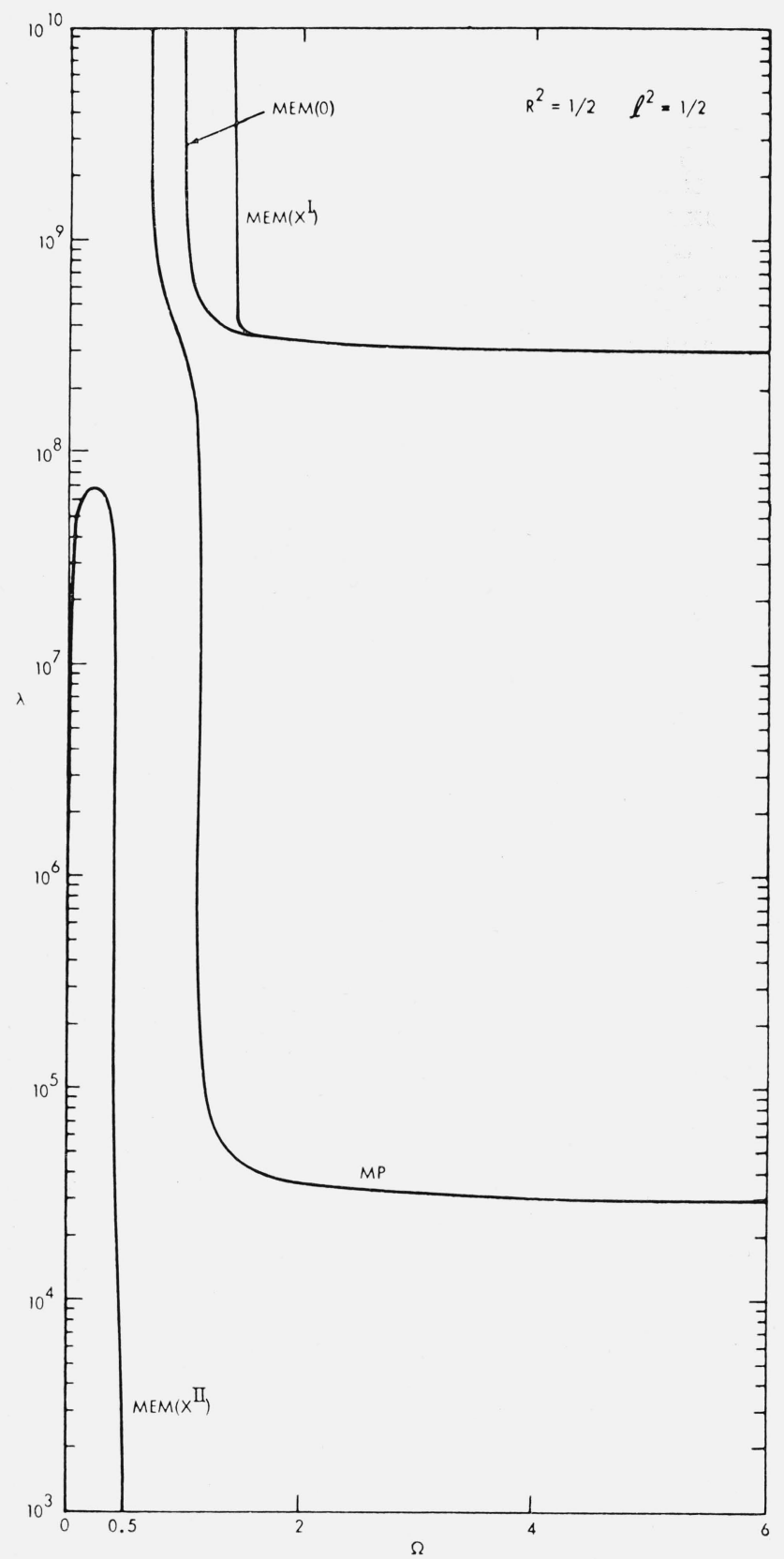

Figure 7a. Complete dispersion curves for the arbitrary direction of propagation $\left(\mathrm{R}^{2}=1 / 2, l^{2}=1 / 2\right)$. cutoff for $\Omega=1$, asymptotically attains the velocity $C_{0}$ of the electromagnetic waves in free space and has always a phase velocity greater than $C_{0}$. The modified $e^{\mathrm{II}}$ mode is redesignated as the modified plasma mode (MP) since its phase velocity asymptotically approaches the acoustical velocity in the electron gas. The $e^{\mathrm{I}}$ mode is redesigned as the modified extraordinary electromagnetic mode $\left[\mathrm{MEM}\left(X^{\mathrm{I}}\right)\right]$ since its phase velocity also asymptotically approaches $C_{0}$ in the limit of infinite frequency. The modified $O^{\mathrm{II}}$ mode for large values of $R$ may overlap with either the MP or the $\operatorname{MEM}(O)$ modes and therefore, should be another branch of the modified

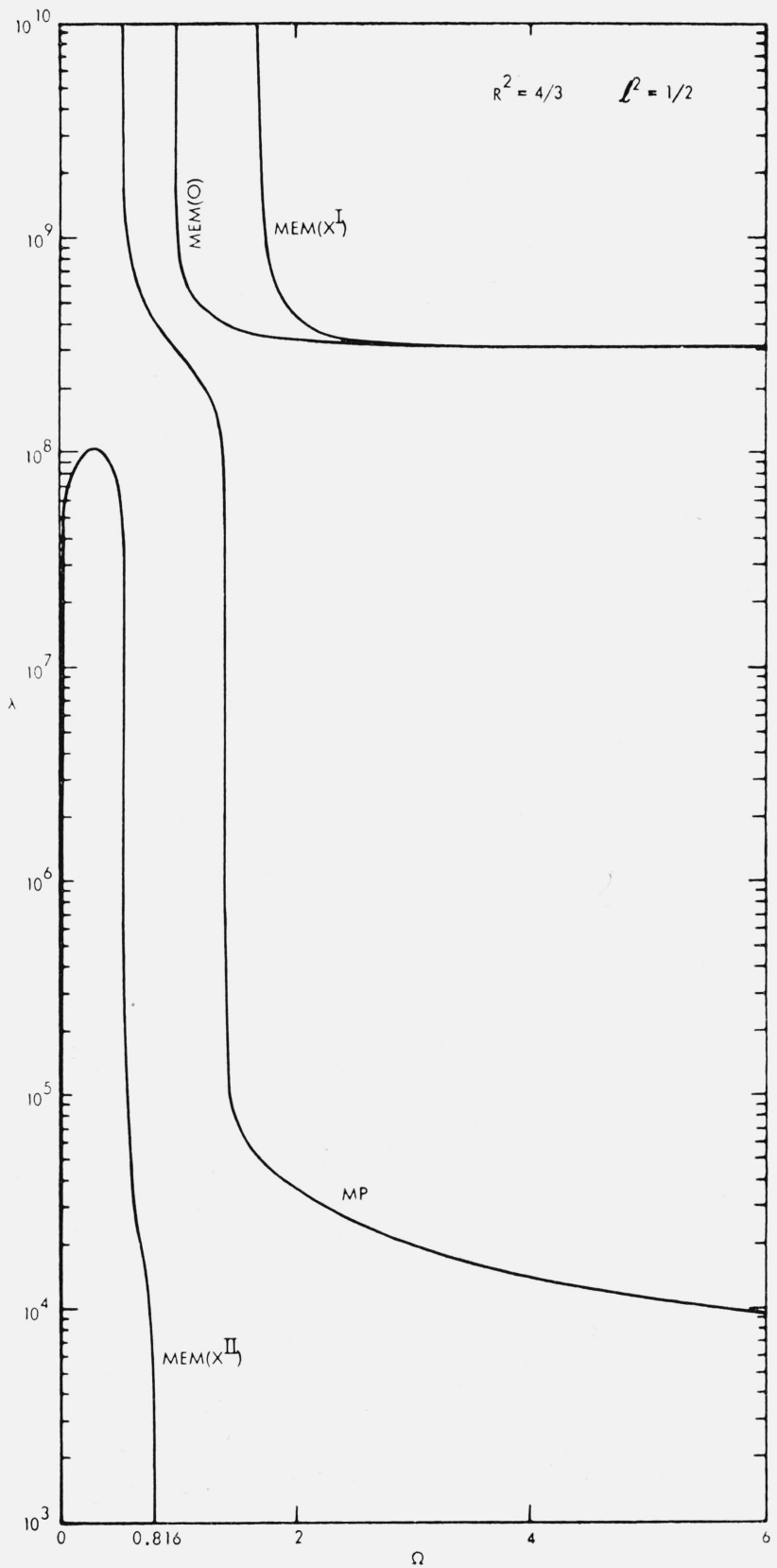

Figure 7b. Complete dispersion curves for the arbitrary direction of propagation $\left(\mathrm{R}^{2}=4 / 3, l^{2}=1 / 2\right)$. 
extraordinary electromagnetic mode $\left[\mathrm{MEM}\left(X^{\mathrm{II}}\right)\right]$. The reason for naming the second modified electromagnetic mode as extraordinary is perhaps due to the fact that it has a branch wherein the phase velocity has considerably lower values than $C_{0}$. Also unlike the other modes, the first part of the $\operatorname{MEM}\left(X^{I I}\right)$ mode has a phase velocity that increases with frequency.

In the absence of the external static magnetic field, the plasma and the electromagnetic modes are uncoupled. The plasma mode is entirely longitudinal and the electromagnetic mode is totally transverse. However in the presence of the static external mag-

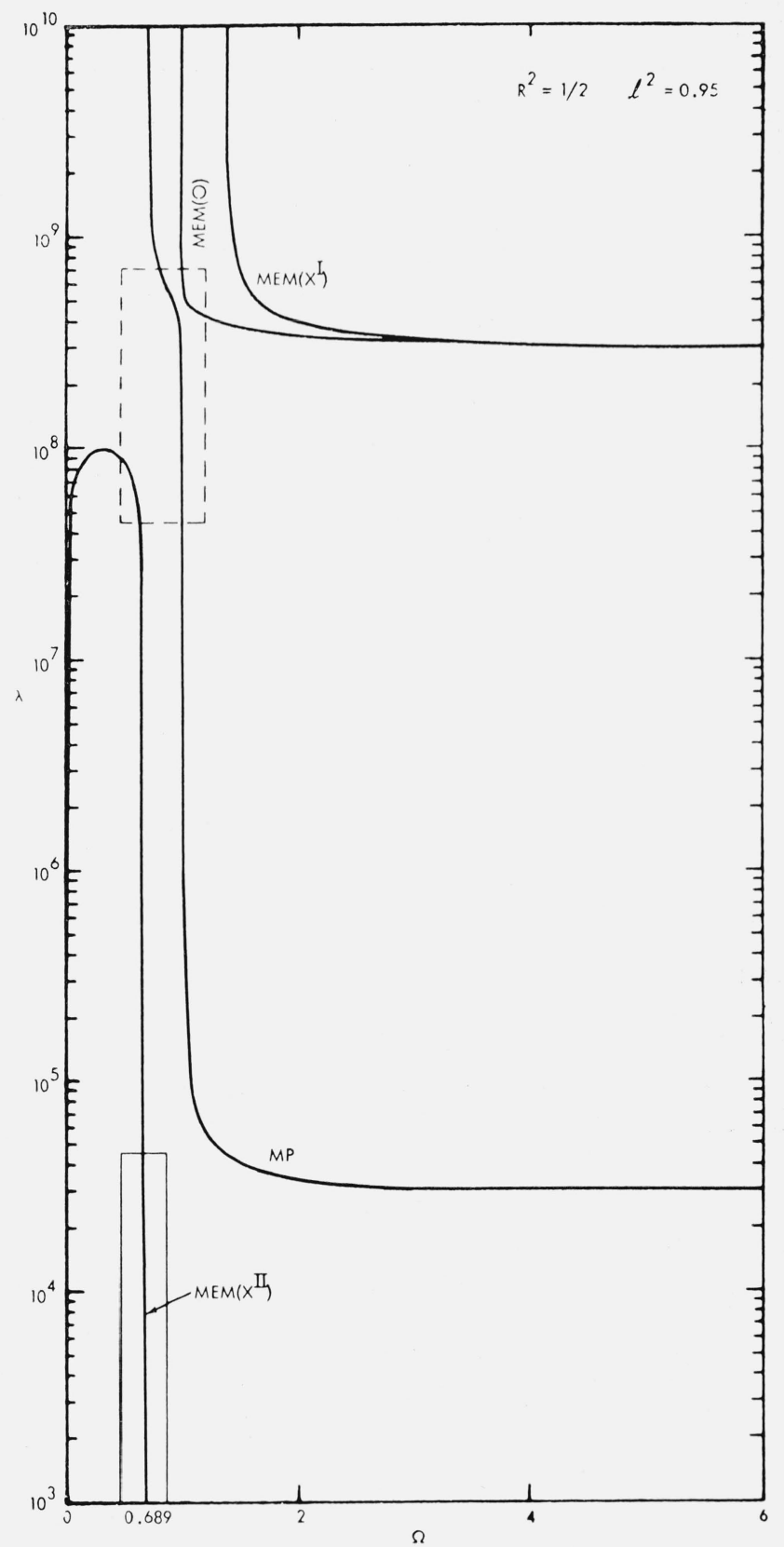

Figure 8a. Complete dispersion curves for the arbitrary direction of propagation $\left(\mathrm{R}^{2}=1 / 2, l^{2}=0.95\right)$. netic field, the modes of isotropic plasma get mixed so that all the modes in an anisotropic plasma have both longitudinal as well as transverse field components. Hence all the three modes in an anisotropic plasma are designated as modified in order to be able to distinguish them from the corresponding modes in an isotropic plasma.

Even though the three modes in an anisotropic plasma have both longitudinal and transverse field components, it is found that each of the modes is predominantly longitudinal in certain ranges of frequency and predominantly transverse in the remaining ranges of frequency. The modified ordinary

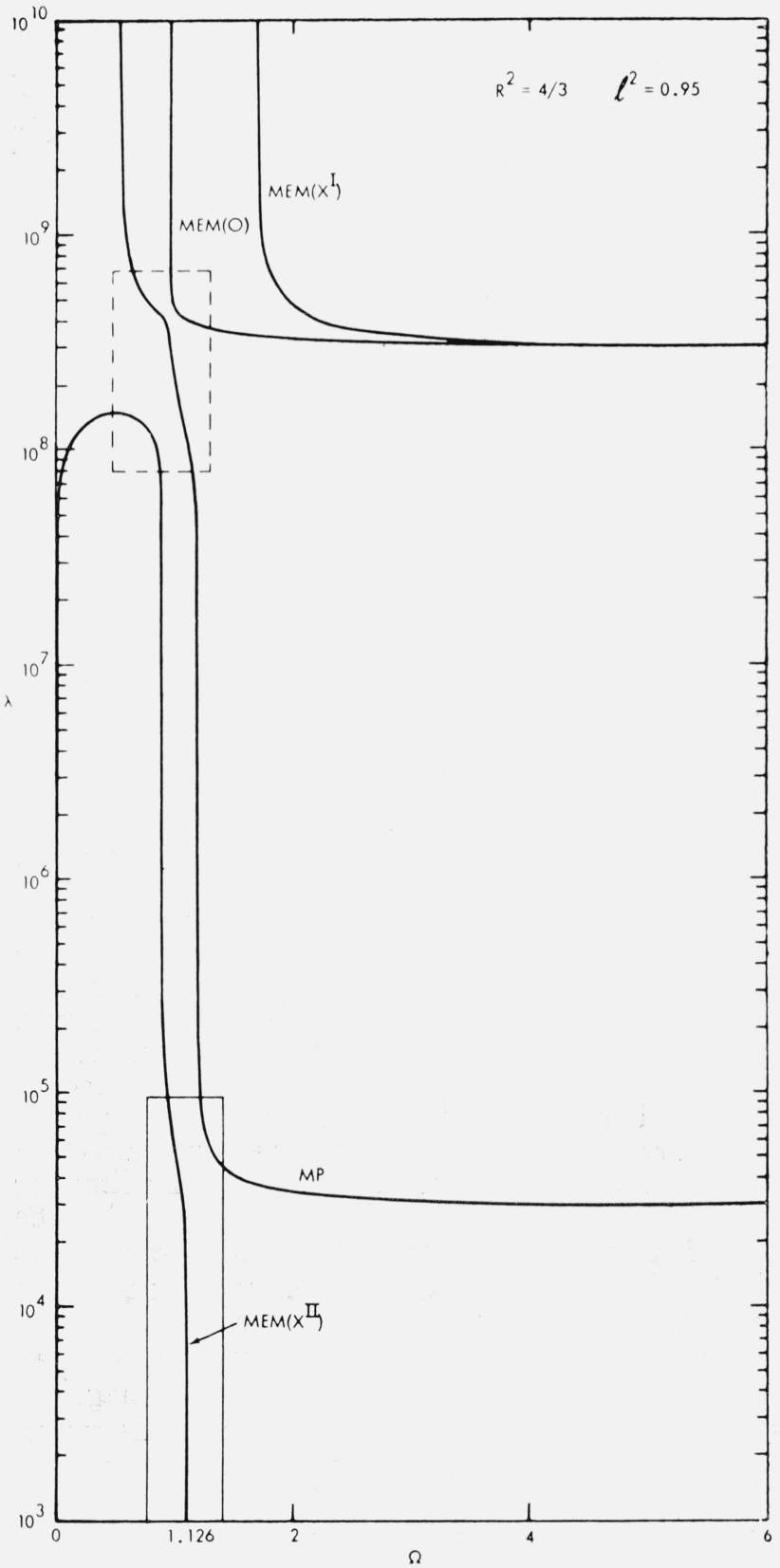

Figure 8b. Complete dispersion curves for the arbitrary direction of propagation $\left(\mathrm{R}^{2}=4 / 3, l^{2}=0.95\right)$. 
electromagnetic mode is predominantly transverse throughout its propagation range $1<\Omega<\infty$. The modified extraordinary electromagnetic mode is predominantly transverse in the frequency ranges $0<\Omega<\Omega_{3}$ and $\Omega_{2}<\Omega<\infty$ and is predominantly longitudinal in the frequency range $\Omega_{3}<\Omega<R l$ and in the neighborhood of $\Omega=\Omega_{3}$, the rapid transition from a transverse to a longitudinal wave takes place. The modified plasma mode is predominantly transverse in the frequency range $\Omega_{1}<\Omega<\Omega_{4}$, rapidly changes character in the neighborhood of the îrequency $\Omega=\Omega_{4}$ to become predominantly longitudinal in the remaining frequency range $\Omega_{4}<\Omega<\infty$.

As the direction of propagation of the plane wave is changed so as to approach the direction perpendicular to the static magnetic field, $l^{2}$ becomes smaller and this has effect only on the values of $\Omega_{3}, R l$ and $\Omega_{4}$. It is found that $l^{2}$ approaches zero both $\Omega_{3}$ and $R l$ tend to zero and $\Omega_{4}$ tends to the value $\sqrt{1+R^{2}}$. It follows therefore that as the propagation direction is changed so as to approach the direction perpendicular to the static magnetic field, the $\operatorname{MEM}(O)$ and the $\operatorname{MEM}\left(X^{\mathrm{I}}\right)$ modes remain practically unaltered whereas the propagation range of the $\operatorname{MEM}\left(X^{\text {II }}\right)$ mode gets continually diminished until it finally vanishes in the limiting case of propagation across the static magnetic field. For the MP mode, the only effect is the frequency $\Omega_{4}$ around which it changes from being predominantly transverse to predominantly longitudinal, continually increases as $l^{2}$ is decreased and approaches the value of $\sqrt{1+R^{2}}$ in the case of propagation across the static magnetic field. A comparison of these results with those obtained for the special case of propagation across the static magnetic field, as depicted in figure $3 \mathrm{~b}$ shows that the dispersion for the three modes obtained here for the case of propagation at an arbitrary angle to the direction of the static magnetic field, attains the proper limiting values for propagation across the static magnetic field.

On the other hand, as the direction of propagation is changed so as to approach that of the static magnetic field, $l^{2}$ increases continually and attains the value unity. As $l^{2}$ increases to the value unity, $R l$ approaches $R$ and $\Omega_{3}$ and $\Omega_{4}$ approach respectively the smaller or the larger of the two values, 1 and $R$. It is found that the dispersion curves for the propagation direction at an arbitrary angle to the direction of the static magnetic field do not in the limiting case of $l^{2}=1$, become identical with those for the case of propagation along the magnetic field as depicted in figure $2 \mathrm{~b}$. Consequently the results of the higher order approximations obtained in this section hold good everywhere except in a small boundary region about the direction of the magnetic field and, therefore, are said to pertain to the region exterior to the axial boundary layer. In the axial boundary layer, the dispersion curves undergo rapid changes due to the decoupling of the plasma mode. A different type of higher order perturbations is therefore called for in the case of propagation very close to the direction of the static magnetic field.

The dispersion curves for the three modes are drawn in figures $8 \mathrm{a}$ and $\mathrm{b}$ for two values of the parameter $R$, namely (i) $R^{2}=1 / 2$ and (ii) $\mathrm{R}^{2}=4 / 3$ for the case in which the direction of propagation is very close to that of the static magnetic field. Specifically the value $l^{2}=0.95$ is used. An examination of figure 8 a shows that for the case $R^{2}<1$, the dispersion curves for the modified plasma mode and the modified ordinary electromagnetic mode approach very close to each other in the neighborhood of the frequency $\Omega=1$. Similarly from figure $8 b$, it is seen that for the case $R^{2}>1$, the dispersion curve for the modified plasma mode approaches both the curves for the modified extraordinary and the modified ordinary electromagnetic modes in the neighborhood of the frequency $\Omega=1$. Also the dispersion curves for the modified plasma mode and the modified extraordinary electromagnetic mode approach each other again in the neighborhood of the frequency $\Omega=R$. It may therefore be anticipated that the dispersion in the axial boundary layer might undergo rapid changes in the neighborhood of the two frequencies $\Omega=R$ and $\Omega=1$.

\section{Dispersion in the Axial Boundary Layer}

For the purpose of obtaining the proper approximations in the axial boundary layer, it is convenient to start by rewriting (12a-d) as follows:

$$
\begin{aligned}
& A_{10}=-C_{0}^{2} \Omega^{2}\left[2\left(\Omega^{2}-1\right)\right.\left(\Omega^{2}-1-R^{2}\right)+R^{2}\left(l^{2}-1\right) \\
&\left.+\frac{a^{2}}{C_{0}^{2}}\left\{\left(\Omega^{2}-1\right)^{2}-\Omega^{2} l^{2} R^{2}\right\}\right] \\
& A_{20}=C_{0}^{4} \Omega^{2}\left[\left(\Omega^{2}-1\right)\left(\Omega^{2}-R^{2}\right)+R^{2}\left(l^{2}-1\right)\right. \\
&\left.+2 \Omega^{2} \frac{a^{2}}{C_{0}^{2}}\left\{\Omega^{2}-1-l^{2} R^{2}\right\}\right]
\end{aligned}
$$

$A_{30}=-C_{0}^{4} a^{2} \Omega^{4}\left[\Omega^{2}-R^{2}+R^{2}\left(1-l^{2}\right)\right]$

and

$A_{0}=\left(\Omega^{2}-1\right)\left[\Omega^{4}-\Omega^{2}\left(2+R^{2}\right)+1\right]$

The axial boundary layer may be easily shown to be defined by those values of $\theta$ for which

$$
l^{2}=1-t \frac{a^{2}}{C_{0}^{2}}
$$

where $t>0$ and is of the order unity. First, it is desired to restrict attention to the neighborhood of the frequency $\Omega=R$. For this purpose, let

$$
\Omega^{2}=R^{2}+s \frac{a^{2}}{C_{0}^{2}}
$$

where $s$ is of the order unity. The substitution of (44) and (45) in (43a-d) and the rearrangement of the terms in powers of $a^{2} / C_{0}^{2}$, yields the following 
$A_{10}=-C_{0}^{2}\left[A_{10}^{(0)}+A_{10}^{(1)} \frac{a^{2}}{C_{0}^{2}}+A_{10}^{(2)} \frac{a^{4}}{C_{0}^{4}}+A_{10}^{(3)} \frac{a^{6}}{C_{0}^{6}}+A_{10}^{(4)} \frac{a^{8}}{C_{0}^{8}}\right]$

(46a)

$A_{20}=C_{0}^{2} a^{2}\left[A_{20}^{(0)}+A_{20}^{(1)} \frac{a^{2}}{C_{0}^{2}}+A_{20}^{(2)} \frac{a^{4}}{C_{0}^{4}}+A_{20}^{(3)} \frac{a^{6}}{C_{0}^{6}}\right]$

$A_{30}=-C_{0}^{2} a^{4}\left[A_{30}^{(0)}+A_{30}^{(1)} \frac{a^{2}}{C_{0}^{2}}+A_{30}^{(2)} \frac{a^{4}}{C_{0}^{4}}\right]$

and

$$
A_{0}=A_{0}^{(0)}+A_{0}^{(1)} \frac{a^{2}}{C_{0}^{2}}+A_{0}^{(2)} \frac{a^{4}}{C_{0}^{4}}+A_{0}^{(3)} \frac{a^{6}}{C_{0}^{6}}
$$

where

$$
\begin{aligned}
& A_{10}^{(0)}=2 R^{2}\left(1-R^{2}\right) \\
& A_{20}^{(0)}=R^{4}(s-t-2)-R^{2} s \\
& A_{30}^{(0)}=R^{6} t+R^{4} s \\
& A_{0}^{(0)}=-2 R^{4}+3 R^{2}-1 .
\end{aligned}
$$

The expressions for all the other $A$ 's are not needed in this analysis and hence are not reproduced here. The exact dispersion equation (11) may be rewritten with the help of (46) and (47) as follows:

$$
\begin{gathered}
{\left[A_{0}^{(0)}+A_{0}^{(1)} \frac{a^{2}}{C_{0}^{2}}+A_{0}^{(2)} \frac{a^{4}}{C_{0}^{4}}+A_{0}^{(3)} \frac{a^{6}}{C_{0}^{6}}\right] \lambda^{6}} \\
-C_{0}^{2}\left[A_{10}^{(0)}+A_{10}^{(1)} \frac{a^{2}}{C_{0}^{2}}+A_{10}^{(2)} \frac{a^{4}}{C_{0}^{4}}+A_{10}^{(3)} \frac{a^{6}}{C_{0}^{6}}+A_{10}^{(4)} \frac{a^{8}}{C_{0}^{8}}\right] \lambda^{4} \\
+C_{0}^{2} x^{2}\left[A_{20}^{(0)}+A_{20}^{(1)} \frac{a^{2}}{C_{0}^{2}}+A_{20}^{(2)} \frac{a^{4}}{C_{0}^{4}}+A_{20}^{(3)} \frac{a^{6}}{C_{0}^{6}}\right] \lambda^{2} \\
-C_{0}^{2} x^{4}\left[A_{30}^{(0)}+A_{30}^{(1)} \frac{a^{2}}{C_{0}^{2}}+A_{30}^{(2)} \frac{a^{4}}{C_{0}^{4}}\right]=0
\end{gathered}
$$

Note that all the $A$ 's are of the order unity.

If the phase velocities of the order $a$ or lower are only sought, it is legitimate to retain on the left side of (48) only the leading terms which are of the order $C_{0}^{2} a^{4}$ and neglect all the other terms which are at least of the order $a^{2} / C_{0}^{2}$ lower than the leading terms, resulting in the following quadratic equation in $\lambda^{2}$ :

$$
\lambda^{4}-a^{2} \frac{A_{20}^{(0)}}{A_{10}^{(0)}} \lambda^{2}+a^{4} \frac{A_{30}^{(0)}}{A_{10}^{(0)}}=0
$$

provided $A_{10}^{(0)} \neq 0$. Using (47) in (49), it is found that

$$
\left(\frac{\lambda}{a}\right)^{4}+2 U\left(\frac{\lambda}{a}\right)^{2}+W=0
$$

where

$$
U=\frac{s\left(R^{2}-1\right)-R^{2}(t+2)}{4\left(R^{2}-1\right)} \quad W=-\frac{R^{2}\left(R^{2} t+s\right)}{2\left(R^{2}-1\right)} .
$$

For $t=0$, which corresponds to the case of propagation along the static magnetic field, the two modes whose phase velocities are specified by (50a) are decoupled as evidenced by the fact that (50a) factors into two linear equations in $\left(\frac{\lambda}{a}\right)^{2}$ yielding the following solutions :

$$
\begin{array}{ll}
\left(\frac{\lambda}{a}\right)_{1}^{2}=-\frac{s}{2} & \text { for } t=0 \\
\left(\frac{\lambda}{a}\right)_{2}^{2}=\frac{R^{2}}{R^{2}-1} & \text { for } t=0 .
\end{array}
$$

For $t \neq 0$, the solutions of (50a) are given by

$$
\left(\frac{\lambda}{a}\right)_{1,2}^{2}=-U \pm \sqrt{U^{2}-W} \quad \text { for } t \neq 0 .
$$

It may be easily shown that $U^{2}-W$ can never vanish, with the result, the two phase velocities specified by (52) are always different.

The phase velocity given by $(51 \mathrm{a}, \mathrm{b})$ and by (52) for $t=1$ are sketched in figures $9 \mathrm{a}$ and $9 \mathrm{~b}$ for (i) $R^{2}<1$ and (ii) $R^{2}>1$, respectively. For $R^{2}<1,\left(\frac{\lambda}{a}\right)_{2}$ does not lead to real phase velocities and $\left(\frac{\lambda}{a}\right)_{1}$ corresponds to real phase velocities for $s<-R^{2} t$. Also $\left(\frac{\lambda_{1}}{a}\right)=0$ for $s=-R^{2} t$. For $R^{2}>1$, (51a) for $s<0$ and (51b) for all $s$ leads to real phase velocities. Also $(\lambda / a)_{1}$ in (52) for all $s$ and $(\lambda / a)_{2}$ for $s<-R^{2} t$ leads to real phase velocities. Also $(\lambda / a)_{1}>(\lambda / a)_{2}$ for $s<-R^{2} t$ and $(\lambda / a)_{2}=0$ for $s=-R^{2} t$. From (44) and (45), it is clear that $s=-R^{2} t$ corresponds to $\Omega=R l$. For $R^{2}>1$ and $t=0$, the two phase velocities are found to be equal for $s=-2 R^{2} / R^{2}-1$.

In the axial boundary layer, in order to understand the physical significance of the variation with respect to $s$ of the phase velocities of the order of the acoustic velocity $a$ in the electron gas or lower, for the frequencies in the close neighborhood of $\Omega=R$, it is necessary to compare figure 9 a with that portion of figures $2 \mathrm{~b}$ and $8 \mathrm{a}$ enclosed by a solid line. The portion of the dispersion curve enclosed by a solid line in figure $8 \mathrm{a}$ is just the modified extraordinary electromagnetic mode, $\operatorname{MEM}\left(X^{\mathrm{II}}\right)$, whose phase velocity, according to (34), goes to zero for $\Omega=R l$. The phase velocity in figure $9 \mathrm{a}$, according to the foregoing discussion, goes to zero for $s=-R^{2} t$, or $\Omega=R l$. Therefore the phase velocity diagram for $t \neq 0$ in figure $9 \mathrm{a}$ is just the expanded version of the dispersion curve of the $\operatorname{MEM}\left(X^{\mathrm{II}}\right)$ mode near $\Omega=R$ and $\lambda=a$. When $t$ approaches zero, figure 9 a shows that the dispersion curve for the MEM $\left(X^{\mathrm{II}}\right)$ mode just shifts to the higher frequency side and has its zero now at $s=0$ or $\Omega=R$. A comparison with figure $2 \mathrm{~b}$ shows that the phase velocity diagram in figure 9a for the for the case $t=0$, is the same as the purely transverse extraordinary electromagnetic mode propagating along the direction of the static magnetic field. 


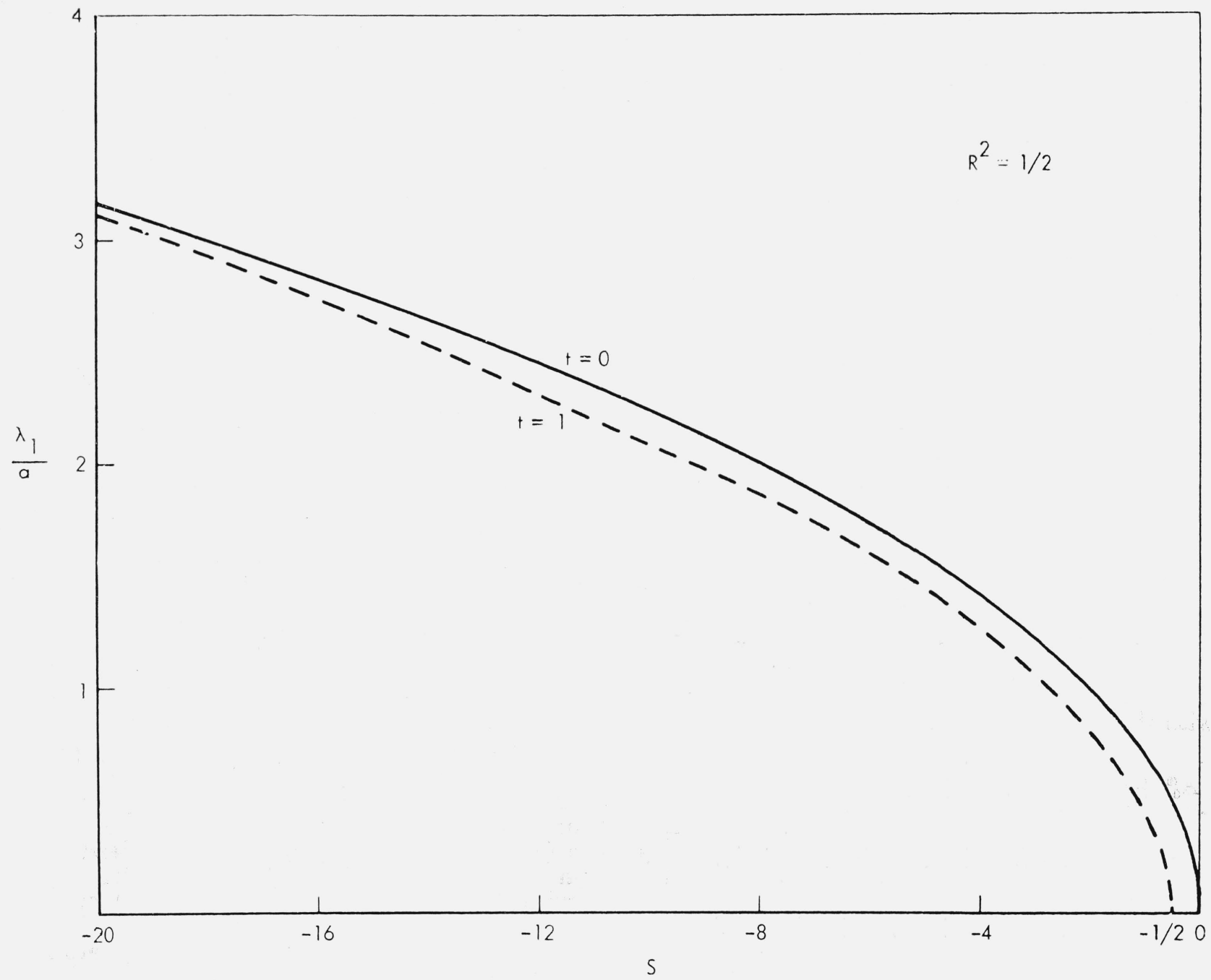

FIGURE 9a. Dispersion in the axial boundary layer in the neighborhood of $\Omega=\mathrm{R}$ and $\lambda=\mathrm{a}\left(\mathrm{R}^{2}=1 / 2\right)$.

Summarizing the above results, it follows that for the case $R^{2} \leq 1$, the modified extraordinary electromagnetic $\left[\operatorname{MEM}\left(X^{\mathrm{II}}\right)\right]$ mode in the neighborhood of $\Omega=R$ continuously goes over to the uncoupled, purely transverse electromagnetic mode as the direction of propagation approaches and finally coincides with that of the static magnetic field.

The portion enclosed by the solid line in figure $2 \mathrm{c}$ has two dispersion curves. The phase velocity curve which goes to zero for $\Omega=R$ belongs to the purely transverse extraordinary electromagnetic mode and the other curve which is nearly horizontal belongs to the purely longitudinal plasma mode. These curves refer to propagation in the direction of the static magnetic field. From (16), the phase velocity of the plasma mode for $\Omega=R$ is seen to be equal to $\frac{a R}{\sqrt{R^{2}-1}}$. There are two intersecting curves for the phase velocity in figure $9 \mathrm{~b}$ for the case $t=0$, which refers to propagation along the static magnetic field.
The curve for $\lambda_{1}$, obviously, is a part of the purely transverse extraordinary electromagnetic mode since it has a value zero for $s=0$ or $\Omega=R$. The other curve, namely that for $\lambda_{2}$, as seen from (51a), has the same phase velocity as that of the plasma mode for $\Omega=R$ and hence is a part of the dispersion curve for the purely longitudinal plasma mode. Note that these two dispersion curves intersect and hence have the same phase velocities for $s=-2 R^{2} / R^{2}-1$. The portion enclosed by the solid line in figure $9 \mathrm{~b}$ has two, nonintersecting dispersion curves. Obviously, the one on the low frequency side is a part of the modified extraordinary electromagnetic mode, since it has zero value for $\Omega=R l$. The other curve, which lies on the higher frequency side, is a part of the modified plasma mode. These two nonintersecting dispersion cuives refer to propagation in a direction which makes a small angle with that of the static magnetic field. On comparing the two dispersion curves enclosed by the solid line with the two curves in figure $9 \mathrm{~b}$ for the case $t \neq 0$, it is evident that the 


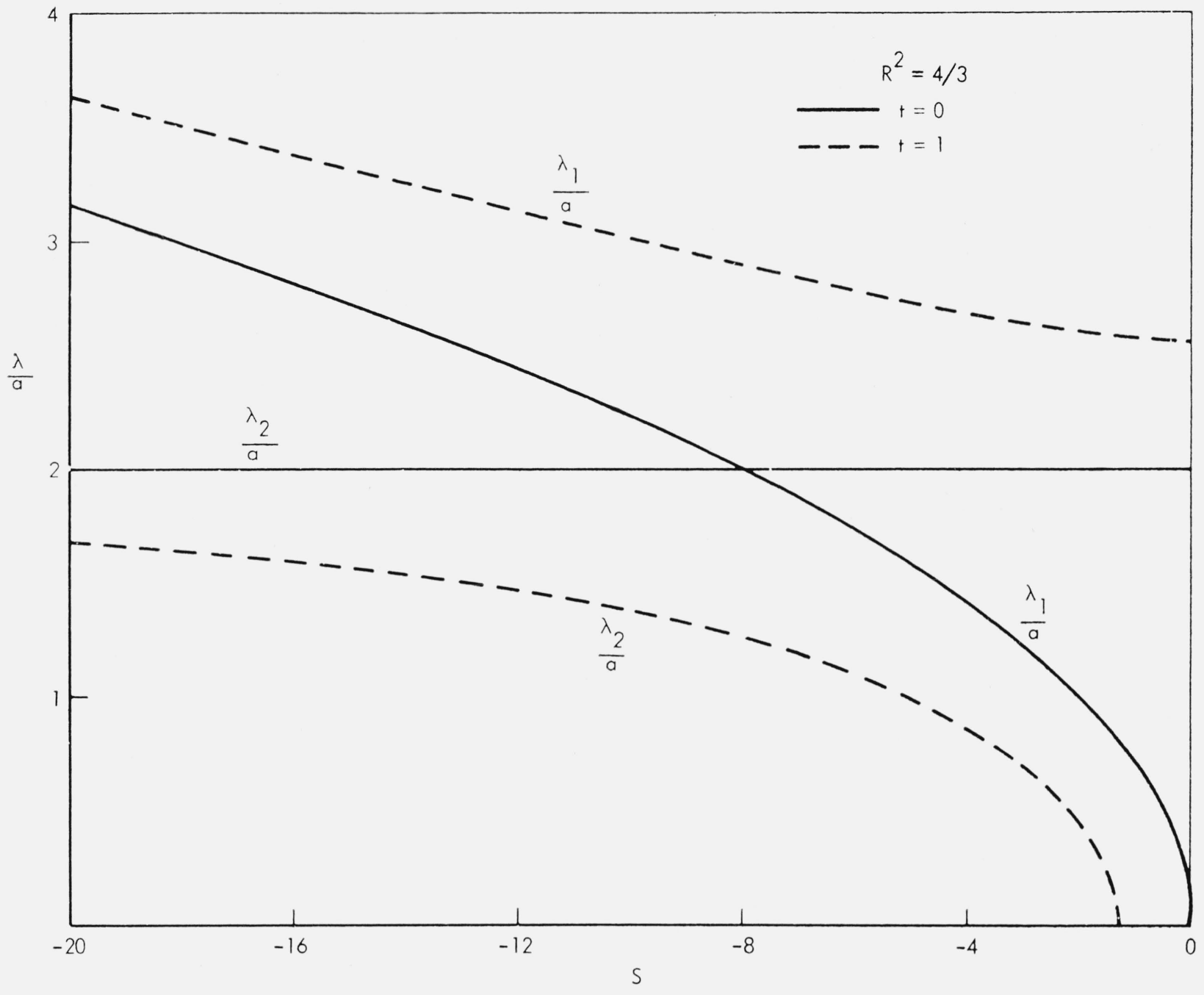

FIGURE 9b. Dispersion in the axial boundary layer in the neighborhood of $\Omega=\mathrm{R}$ and $\lambda=\mathrm{a}\left(\mathrm{R}^{2}=4 / 3\right)$.

phase velocity curves for $\lambda_{1}$ and $\lambda_{2}$ belong to the modified plasma mode and the modified extraordinary electromagnetic mode $\left[\operatorname{MEM}\left(X^{\mathrm{II}}\right)\right]$ respectively. The results depicted in figure $9 \mathrm{~b}$ show the transition that takes place in the axial boundary layer for the case $R^{2}>1$. From an examination of figure $9 \mathrm{~b}$, it is clear that as the direction of propagation approaches and finally reaches that of the static magnetic field, the higher frequency side of the dispersion curve for the modified plasma mode merges with the lower frequency side of the dispersion curve for the $\operatorname{MEM}\left(X^{I I}\right)$ mode to form a continuous curve which belongs to the uncoupled, purely longitudinal plasma mode. On the other hand, the higher frequency side of the dispersion curve of the $\mathrm{MEM}\left(X^{\mathrm{II}}\right)$ mode crosses that of the uncoupled plasma mode, merges with the lower frequency branch of the modified plasma mode to form a continuous dispersion curve which obviously belongs to the uncoupled, purely transverse extraordinary electromagnetic mode. Simple evaluation of the generalized Poynting vector may be made to establish that the two modes whose dispersion curves cross each other for $t=0$ near $\Omega=R$ and $\lambda=a$ are uncoupled. Thus in the axial boundary layer, for the case $R^{2}>1$, a part of the decoupling of the plasma mode takes place in the close neighborhood of $\Omega=R$ and $\lambda=a$.

It is now desired to examine the behavior of the dispersion in the neighborhood of the frequency $\Omega=1$. For this purpose, let

$$
\Omega^{2}=1+s \frac{a^{2}}{C_{0}^{2}}
$$

where $s$ is of the order unity. Employing the same procedure as before and making use of (44) and (53), it may be easily derived that the phase velocities of the order $C_{0}$ or higher are specified by the following cubic equation in $\lambda^{2}$ : 


$$
\begin{aligned}
\left(\frac{\lambda}{C_{0}}\right)^{6}- & \frac{(1+t+2 s)}{s}\left(\frac{\lambda}{C_{0}}\right)^{4} \\
& \quad-\frac{s-R^{2}(s+t+2)}{R^{2} s}\left(\frac{\lambda}{C_{0}}\right)^{2}+\frac{\left(-R^{2}+1\right)}{R^{2} s}=0 .
\end{aligned}
$$

For $t=0$, which corresponds to the case of propagation along the static magnetic field, the three modes whose phase velocities are specified by (54), are decoupled as evidenced by the fact that (54) factors into three linear equations in $(\lambda / a)^{2}$ yielding the following solutions:

$$
\begin{aligned}
& \left(\frac{\lambda_{1}}{C_{0}}\right)^{2}=\frac{1}{s} \quad \text { for } t=0 \\
& \left(\frac{\lambda_{2}}{C_{0}}\right)^{2}=\frac{R+1}{R} \quad \text { for } t=0
\end{aligned}
$$

and

$$
\left(\frac{\lambda_{3}}{C_{0}}\right)^{2}=\frac{R-1}{R} \quad \text { for } t=0 \text {. }
$$

For the case $t \neq 0$, it may be verified that the discriminant of the cubic equation (54) in $\left(\lambda / C_{0}\right)^{2}$ is negative and as a result all the three roots are real and unequal.

The phase velocities given by (54) for $t=1$ and those given by (55) are plotted in figures $10 \mathrm{a}$ and $\mathrm{b}$ for the case (i) $R^{2}<1$ and (ii) $R^{2}>1$, respectively. For $R^{2}<1$, (55a) for $s>0$ and (55b) yield real phase velocities whereas $(55 \mathrm{c})$ does not give rise to real phase velocities. Also, it may be proved that the number of positive roots of (54) is either one or two according as $s$ is less than or greater than zero. Note that only positive real roots will yield real values for the phase velocity. For $R^{2}>1$ (55a) for $s>0,(55 \mathrm{~b})$ and $(55 \mathrm{c})$ yield real phase velocities.

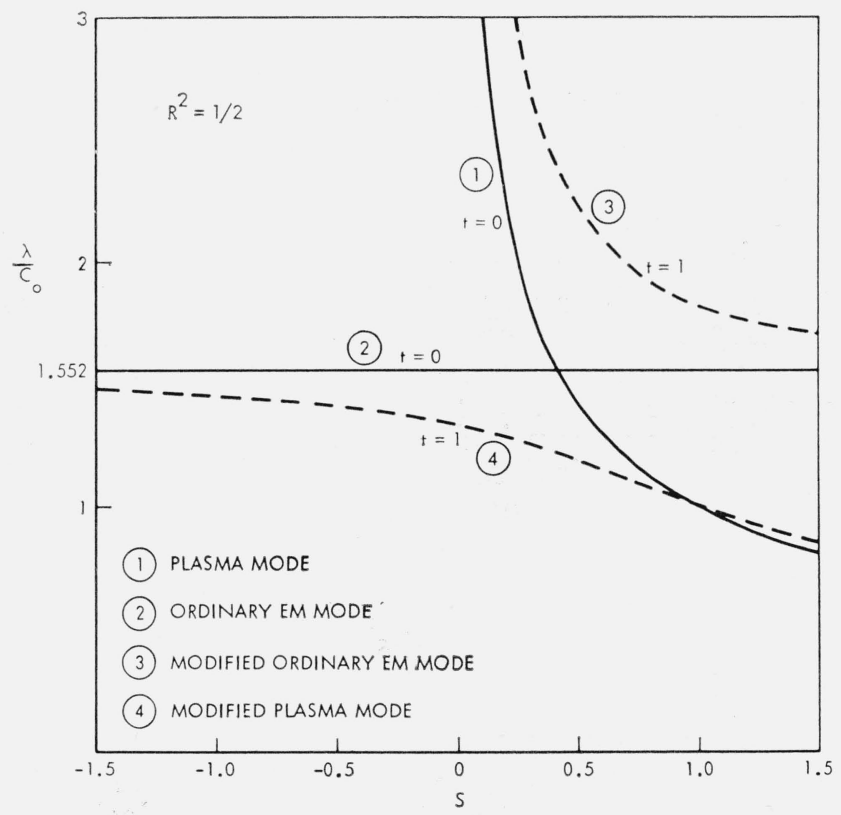

Figure 10a. Dispersion in the axial boundary layer in the neighborhood of $\Omega=1$ and $\lambda=\mathrm{C}_{0}\left(\mathrm{R}^{2}=1 / 2\right)$.
Also the number of positive real roots of (54) giving rise to real phase velocities is either two or three depending on whether $s$ is negative or positive.

For $R^{2}<1$, a comparison of figure 10a with those portions of figures $2 \mathrm{~b}$ and $8 \mathrm{a}$ enclosed by a broken line shows that for the case $t=0$, which corresponds to propagation parallel to the static magnetic field, the curves marked 1 and 2 in figure 10a are the portions of the dispersion curves for the purely longitudinal plasma (P) mode and the entirely transverse ordinary electromagnetic (EM) mode respectively. The phase velocities of these two modes are equal for $s=R / R+1$. Similarly for $t=1$, which corresponds to a direction of propagation very slightly inclined to that of the static magnetic field, the curves labeled 3 and 4 in figure $10 \mathrm{a}$ are the sections of the dispersion curves belonging to the modified ordinary electromagnetic mode and the modified plasma mode respectively. It follows then from figure 10a that as the direction of propagation approaches and finally coincides with that of the static magnetic field, the branch of the modified ordinary electromagnetic mode for $s>R / R+1$ joins with that of the modified plasma mode for $s<R / R+1$ to form a continuous curve which belongs to the uncoupled, purely transverse, ordinary electromagnetic mode and the branch of the modified $P$ mode for $s>R / R+1$ joins with that of the modified ordinary EM mode for $s<R / R+1$ to form a smooth curve which is part of the dispersion curve of the uncoupled, purely longitudinal plasma mode. It may be easily verified that $\lambda / C_{0}=1$ is a solution of (54) for $s=1$ for any value of $R^{2}$ or $t$. From this fact, it is evident that the modified plasma mode has its phase velocity equal to the free space velocity of electromagnetic waves for the frequency given by $\Omega^{2}=1+$ $a^{2} / C_{0}^{2}$.

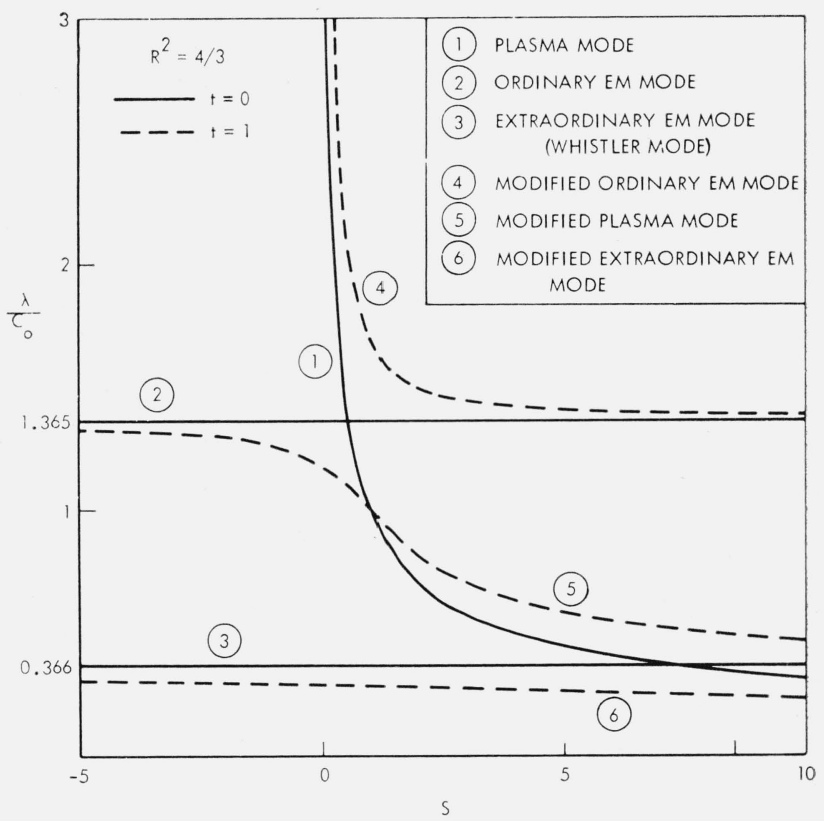

Figure 10b. Dispersion in the axial boundary layer in the neighborhood of $\Omega=1$ and $\lambda=\mathrm{C}_{0}\left(\mathrm{R}^{2}=4 / 3\right)$. 
For the case $R^{2}>1$, a comparison of figure $10 \mathrm{~b}$ with that portion of figures $2 \mathrm{c}$ and $8 \mathrm{~b}$ enclosed by a broken line enables the understanding of mechanism of coupling of the various modes in the axial boundary layer in the close neighborhood of $\Omega=1$. From figure $10 \mathrm{~b}$ it is seen that as the propagation direction is changed from that of the static magnetic field, the branch of the dispersion curve for the plasma mode for $s<R / R+1$ joins with that of the ordinary EM mode for $s>R / R+1$ to form a smooth curve which belongs to the modified ordinary EM mode. And the branch of the dispersion curve of the ordinary EM mode for $s<R / R+1$ joins with that of the plasma mode for $s>R / R+1$ and which in turn merges with that portion of the dispersion curve of the extraordinary EM mode for $s>R / R-1$ to form the dispersion curve corresponding to the modified plasma mode. In a similar manner the branch of the dispersion curve of the extraordinary EM mode for $s<R / R-1$ merges with that of the plasma mode for $s>R / R-1$ to form a smooth dispersion curve belonging to the modified extraordinary EM mode. From the foregoing discussion, the manner in which the plasma mode gets decoupled in the axial boundary layer in the close neighborhood of $\Omega=1$ and $\lambda=C_{0}$, is made clear.

With the understanding of the behavior of the dispersion of the three modes in the axial boundary layer, it is evident that the whistler mode corresponds to the modified extraordinary electromagnetic mode for all directions of propagation and no inconsistency in the nomenclature of the whistler mode arises as in the magneto-ionic approximation.

\section{Concluding Remarks}

It is appropriate to make the following remarks in conclusion. The diagrammatic representation in the $\Omega^{2}-R^{2}$ space used in this paper for elucidating the regions of propagation of the various modes is very similar to the one first introduced by Clemmow and Mullaly [1955] and extensively used by Allis, Buchsbaum, and Bers [1962]. The two parameters used by these authors both contain the source frequency. Only one of the two parameters used in the representation of the parameter space employed in this paper, contains the source frequency and this feature facilitates greatly the determination of the propagation characteristics explicitly as a function of frequency.

The simple explanation that the compressibility of the plasma merely introduces a lower cutoff in the phase velocity curves obtained as a result of the magneto-ionic approximation, is frequently advanced. From the results of this paper, the magneto-ionic theory is seen to yield zero phase velocity for the three frequencies, $\Omega=0, \Omega_{3}$ and $\Omega_{4}$. The compressibility of the plasma does not affect the phase velocity near $\Omega=0$ and merely shifts the zero near $\Omega_{3}$ to the high frequency side with the result that the zero phase velocity now occurs at $\Omega=R l$. Only the phase velocity near $\Omega=\Omega_{4}$, instead of going to zero has a cutoff value equal to the acoustical velocity in the electron gas. Consequently the frequently advanced simple explantion of the effect of compressibility is not fully correct.

Kieburtz [1963] has investigated the same problem that is treated in this paper using the coupled-mode theory and has found, for the range of frequencies given by $\Omega \ll R<1$ in which the whistler phenomenon is observed, that there is coupling between the extraordinary electromagnetic mode (whistler mode) and the purely longitudinal plasma wave. The results obtained in this paper show that for $R<1$, there is no coupling between the whistler mode and the purely longitudinal plasma wave. Therefore, the results reported by Kieburtz appear to be incorrect.

The frequency $\Omega_{4}$, in the close neighborhood of which there is a sharp transition of the character of the wave from being predominantly transverse to being predominantly longitudinal is referred to in the literature [Allis, Buchsbaum, and Bers, 1962] as the frequency of plasma resonance. The results of this investigation show that for $R<1$, the frequency of the plasma resonance is equal to the plasma frequency $\omega_{p}$ in the direction of the static magnetic field and increases gradually to the value $\sqrt{\omega_{p}^{2}+\omega_{c}^{2}}$ for the propagation across the static magnetic field. On the other hand, for $R>1$, the frequency of the plasma resonance is again equal to the plasma frequency $\omega_{p}$ for propagation along the static magnetic field, "jumps" to the value of the gyromagnetic frequency $\omega_{c}$ as soon as the propagation direction is changed ever so little from that of the static magnetic field and increases gradually and attains the same value $\sqrt{\omega_{p}^{2}+\omega_{c}^{2}}$ for propagation across the static magnetic field.

An important and a satisfying result of this investigation is that the magneto-ionic theory is surprisingly accurate over a wide range of the parameters of interest. Where it fails and where inconsistencies set in as a result of its application, the present theory may be used to obviate the difficulties.

The author is grateful to Ronald V. Row for the many valuable discussions and to $\mathrm{N}$. Ciccia for the assistance with the numerical computations.

\section{References}

Allis, W. P., S. J. Buchsbaum, and A. Bers (1962), Waves in anisotropic plasmas, MIT Press Cambridge, Mass.).

Clemmow, P. C., and R. F. Mullaly (1955), Dependence of the refractive index in magnetoionic theory on the direction of the wave normal, Physics of the Ionosphere, Report of Phys. Soc. Conf. Cavendish Lab. p. 340, Physical Society, London.

Kieburtz, R. B. (1963), VLF propagation in a compressible ionosphere, presented at the 1963 PTGAP International Symposium on Space Telecommunications held at the National Bureau of Standards, Boulder, Colo., July 9-11.

(Paper 68D12-434) 